\title{
Outbreak science: recent progress in the detection and response to outbreaks of infectious diseases
}

\author{
Authors: Catherine F Houlihan ${ }^{A}$ and James AG Whitworth ${ }^{B}$
}

The frequency of reported outbreaks of infectious diseases has increased over the past 3 decades, with predictions that this rise will continue. Outbreak response continues to follow nine basic principles: establish the presence of an outbreak, verify the diagnosis, make a case definition, find cases and contacts, conduct basic epidemiology, test hypotheses, institute control measures, communicate the situation and establish ongoing surveillance. Within each of these areas, significant advances have been made over the past 5 years using progress in digital, laboratory, epidemiology and anthropological equipment or techniques. Irrespective of these, future outbreaks of highconsequence are inevitable, and vigilance and preparation must continue in order to prevent significant mortality, morbidity and socio-economic crisis.

\section{Background}

Large recent outbreaks of highly pathogenic or highly transmissible infectious diseases include plague (Madagascar), diphtheria (Bangladesh), Ebola (West Africa (WA) and Democratic Republic of Congo (DRC)), monkeypox (Nigeria), Zika (South and Central America), middle east respiratory syndrome coronavirus (MERS-CoV; Saudi Arabia and Korea) and Lassa fever (LF; Nigeria). Outbreaks of more familiar diseases such as chikungunya, cholera, polio, measles and seasonal influenza have also occurred. The number of reported outbreaks has increased over the past 3 decades. This trend is expected to continue with further zoonotic spill-over events occurring due to population expansion and movement into previously uninhabited regions and the influence of climate change on vector distribution.

Outbreak response continues to follow nine established epidemiological principles. We summarise progress made in these steps using examples of recent outbreaks and future possibilities in Fig 1.

Authors: ${ }^{\text {A }}$ linical lecturer in infectious diseases and virology, Department of Infection and Immunity, University College London and Clinical Research Department, London School of Hygiene and Tropical Medicine, London, UK; ${ }^{B}$ professor of international public health, Department of Infectious Disease Epidemiology, London School of Hygiene and Tropical Medicine, London, UK

\section{Establishing the presence of an outbreak (and} surveillance systems)

An outbreak is defined as more cases of a disease than expected in a specific location over a specific time period. Suspicion often arises when health care workers report an unusual cluster or a single, unexpected presentation. This passive surveillance leads to a delay in the detection of an outbreak. Various efforts have been made to improve this; monitoring social media and internet searches of symptoms have been used detect and report influenza epidemics. ${ }^{1}$ Online platforms have been used to rapidly share or access information about potential outbreaks for 2 decades (ProMED-mail and Global Public Health Intelligence Network), and newer tools have emerged, for example HealthMap, an app which aggregates formal notifications, online news and eyewitness reports. ${ }^{2}$ The World Health Organization's (WHO's) Early Warning and Response System (EWARS) provides a box of electronic equipment, allowing a surveillance system to be deployed rapidly during a humanitarian crisis or outbreak. In rural parts of subSaharan Africa, a portable device is used by minimally-trained

\section{Key points}

The frequency of outbreaks of highly contagious or highly pathogenic diseases is increasing

Surveillance methods now include both digital media and animal surveillance, and electronic tools are increasingly used on the ground to report and monitor outbreaks

Mathematical modelling of outbreaks and molecular sequencing of pathogens have become key components of outbreak response

An ethical framework now exists for the testing of unproven therapies during outbreaks

Anthropologists, social scientists and communication experts are now considered essential members of an outbreak response team

KEYWORDS: Outbreak, global health 


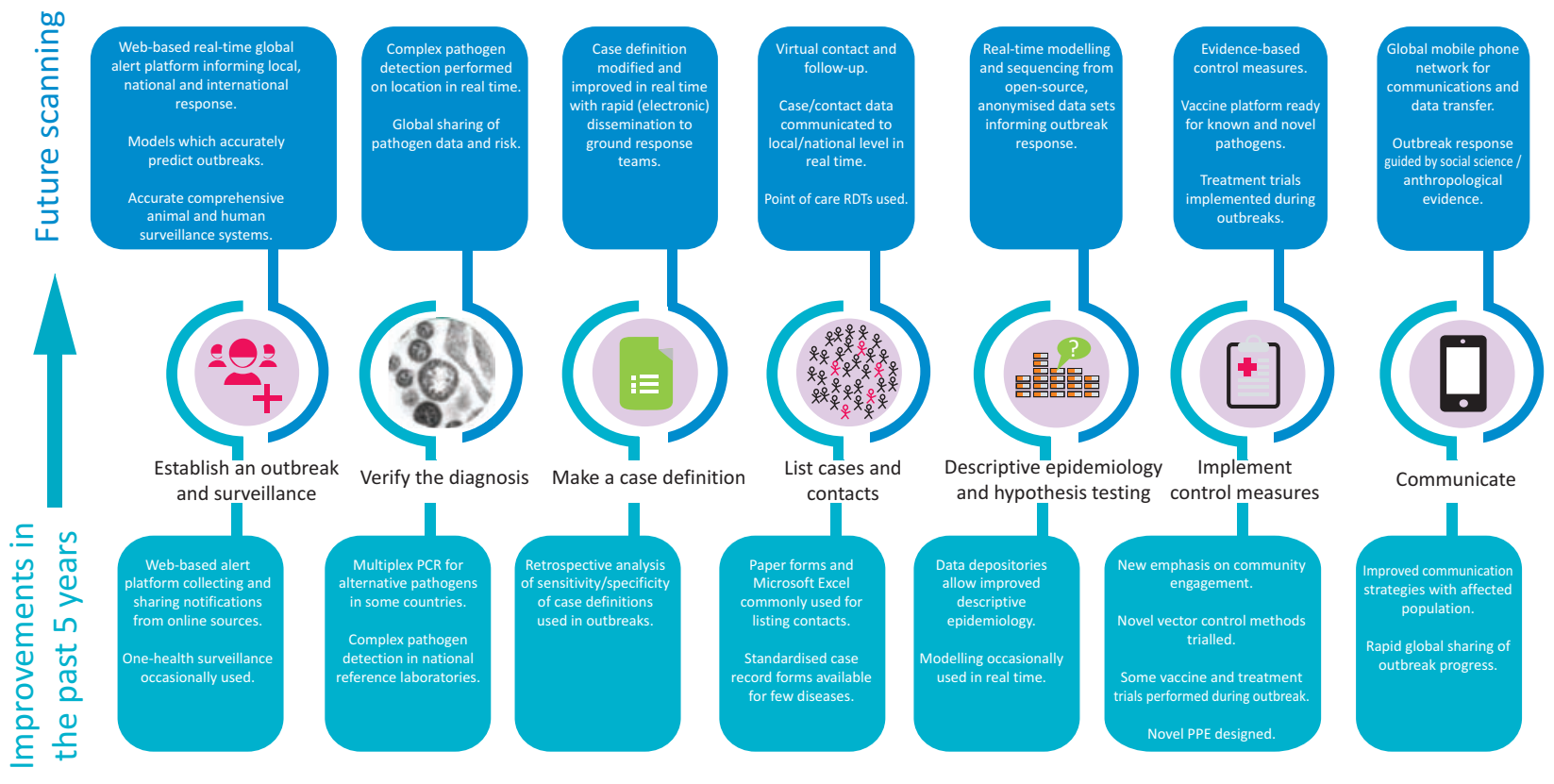

Fig 1. Condensed summary of the epidemiology principles for outbreak response summarising scientific progress made in the recent past (below in teal) and future possibilities (above in blue). PCR = polymerase chain reaction; PPE = personal protective equipment; RDT = rapid diagnostic test (should be sensitive, specific, heat-stable, cheap, simple to use, electricity-free and disposable). Note: the nine outbreak steps have been condensed to seven for space.

surveillance officers to send encrypted images of patients with suspected acute flaccid paralysis, aiding the identification of polio cases. ${ }^{3}$ Importantly, surveillance now includes animals, an approach consistent with 'One Health', for example, surveillance by Public Health England allowed the detection in the Thames estuary of Culex modestus, a mosquito capable of transmitting the West Nile virus.

\section{Verify the diagnosis}

Pathogen identification commonly relies on the transport of samples to reference laboratories with a typical reporting delay of 2-3 days. Suspicion must be maintained that an outbreak may be due to non-infectious causes such as poisoning or nutritional deficiencies. Metagenomic techniques, involving amplifying and sequencing all ribonucleic acid and deoxyribonucleic acid in a sample, is increasingly used to identify new pathogens when standard diagnostic tests are unforthcoming. Metagenomics led to the identification of previously unknown viruses causing haemorrhagic fever including Bas Congo and Lujo. 5,6

\section{Make a case definition}

Defining cases is notoriously challenging during outbreaks when symptoms are non-specific and mimic other diseases. Broad case definitions are used to ensure cases are not missed, but as the 2017 plague epidemic in Madagascar demonstrated, the sheer number of suspect cases can overwhelm control efforts. Progress has been made through real-time analysis of case definitions. In WA in 2015, the sensitivity of the case definition for Ebola was increased from $69 \%$ to over $90 \%$.

\section{Finding cases}

Currently, most national and international outbreak response groups use paper forms for reporting. During recent outbreaks, the use of mobile technology has facilitated the listing, location (using Global Positioning System) and subsequent follow up of cases and contacts using intuitive electronic data-entry platforms. These allow information to be collected in areas without mobile reception, and automatic upload when back online. This mobile technology has assisted in recent outbreaks in the rapid isolation of symptomatic contacts, potentially reducing onward transmission, and allowing improved real-time resource planning. ${ }^{8-11}$

The International Severe Acute Respiratory and Emerging Infection Consortium has generated an open-access portfolio of case record forms for several outbreak diseases and syndromes (https://isaric.tghn.org/protocols) with the aim of standardising clinical and epidemiological information collected during outbreaks.

\section{Rapid diagnostic tests}

The size of an outbreak can be substantially reduced by quick identification of cases using rapid diagnostic tests (RDTs). Three RDTs were validated during the WA Ebola outbreak; some do not require electricity or a high degree of training. ${ }^{12-14}$ GeneXpert, although requiring a power supply, has $>95 \%$ sensitivity and specificity, and is currently used in the DRC for diagnosis and patient discharge. ${ }^{12}$ On the other hand, during the 2017 plague outbreak in Madagascar, an antigen-based RDT had limited specificity and its utility in the response has been questioned. ${ }^{15}$ 


\section{Health systems}

Weak health systems and widespread poverty are key risk factors for outbreaks. Therefore, provision of adequate resources (human, laboratory, logistics and materials) are critical components of every response. Recently, WHO has created a minimum set of standards for accreditation of foreign medical teams, allowing $\mathrm{WHO}$ to coordinate complementary groups to respond, as evidenced in the Bangladesh diphtheria outbreak. Countries such as Sierra Leone have capitalised on international assistance to strengthen their health system after an epidemic, improving primary health care provision, laboratory systems, and specialist diagnostics and treatment. ${ }^{16}$

\section{Descriptive epidemiology and hypothesis testing}

Over the past decade, there has been a movement towards rapid sharing of anonymised outbreak data to strengthen the power of findings from small cohort studies, test hypotheses, develop standards for clinical trials and direct research priorities. This is now a common requirement of journals and funders.
During the WA Ebola outbreak, mathematical modelling was used to predict the rate of increase of the epidemic, and the resources required for an adequate response. ${ }^{17}$ The contribution of risk factors was modelled, allowing targeted interventions like safe burial to gain more focus than, for example, border restrictions. ${ }^{18}$ During the Zika outbreak in Brazil, modelling aided understanding the effect of rising herd immunity on the epidemic's trajectory. ${ }^{19}$ However, accuracy of the models depends on high-quality epidemiological data, which are often lacking.

Hypothesis testing during outbreaks has evolved from attempting to confirm the origin of an outbreak by adding data points to a map of cases (a procedure pioneered by John Snow during the 1854 London cholera outbreak; Fig 2a), to bioinformatic interrogation of the pathogen's genomic data over time and space (Fig 2c). Technology for sequencing of viral and bacterial genomes has advanced significantly; sequencing can now be performed and reported in the field. ${ }^{20}$ During the WA Ebola outbreak, whole genome sequencing identified the origin and spread of the virus with reasonable certainty (Fig $2 b$ ). It also identified episodes of sexual transmission, effective control measures and provided
Fig 2. (a) Map of cholera cases in Soho district of London in 1854 illustrating clustering of cases around the Broad Street pump. (b) Spread of Ebola through west Africa over time in 2014-15 based on phylogenetic tree (in Fig 2c). Lineage $A$ is the initial focus of the outbreak from 2014. Lineage B emerged from A in 2014-15 and spread into Sierra Leone, Liberia and further into Guinea. EBOV entered Mali from two separate routes. (c) Phylogenetic probability tree illustrating EBOV lineages A and $B$ as they emerge over time and geographical location in west Africa. Reproduced with permission from www.nature.com/articles/ nature14594
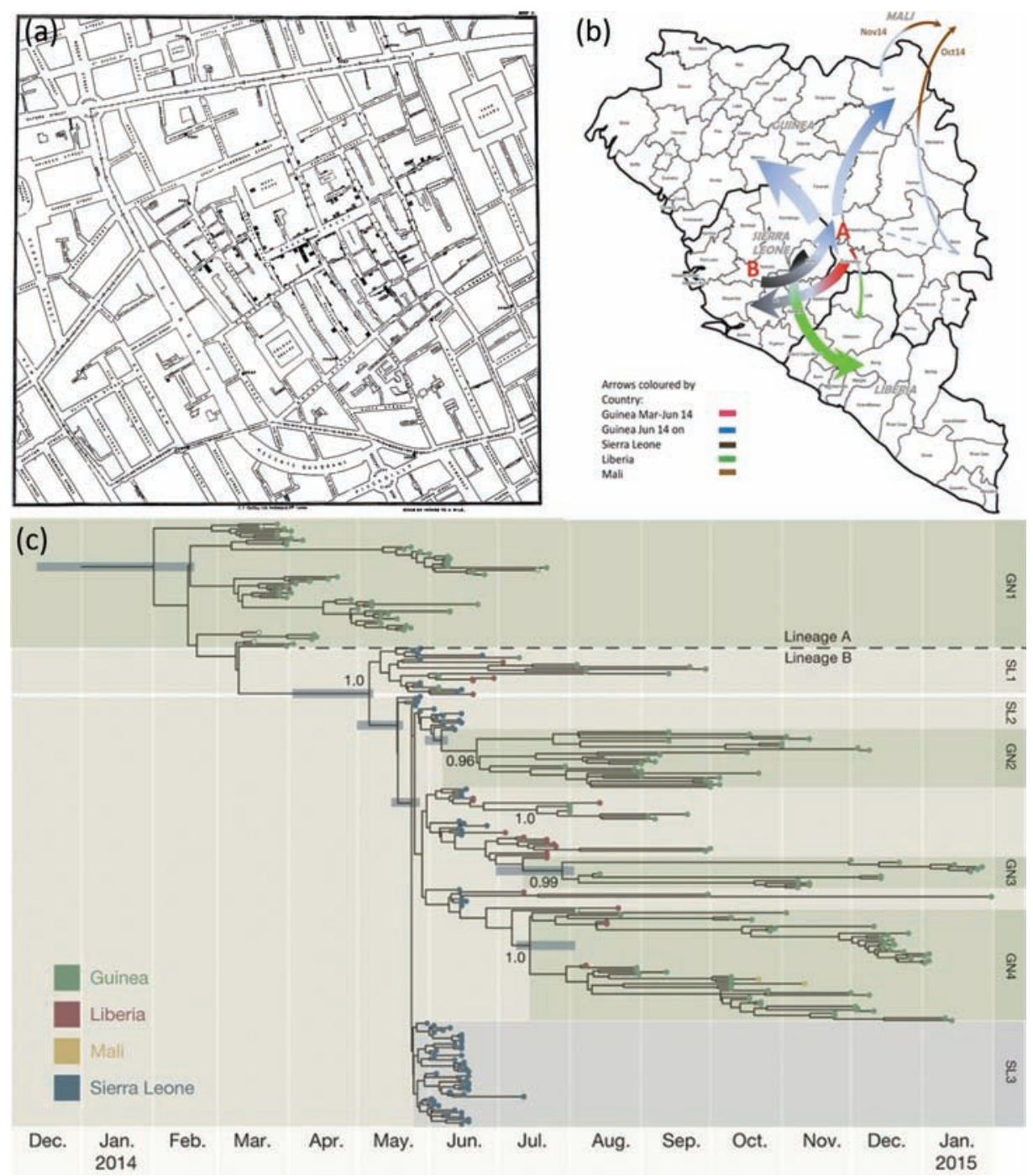
important details for vaccine and therapeutic design. 'Live' maps which document outbreak movement are now available for multiple outbreaks, created using openly available pathogen sequences (www.youtube.com/watch?v=j4Ut4krp8GQ). During the Zika outbreak in Brazil, sequencing was performed using the Oxford-Nanopore MinION, a credit card sized device powered through a laptop USB connection. ${ }^{20}$ Remaining challenges include the sequence accuracy from portable devices, cost and datasharing agreements.

\section{Control measures and communication}

Community engagement is increasingly recognised as a key component of outbreak response, allowing responders to engage the affected population and alter behaviours which may propagate an outbreak. In the Brazil Zika epidemic, messages targeting vector breeding sites were relatively simple. In the WA Ebola outbreak, changing traditional burial practices and isolating sick individuals from family members was more challenging. Anthropologists, communication experts and social scientists are now routinely included in outbreak response teams.

Innovative control measures for arboviruses have been developed. Zika, yellow fever and dengue are transmitted by daybiting mosquitos, meaning that malaria control strategies such as bed nets are less effective. The release of mosquitos infected with the Gram-negative bacteria Wolbachia has significantly reduced vector population size and transmission in experimental settings. ${ }^{21}$

Outbreaks of vaccine-preventable diseases still occur due to a lack of coverage of common childhood vaccines. For most outbreaks (LF, plague, Zika, MERS-CoV, monkeypox, Rift Valley fever) vaccines for human use are only available in trials. Ebola vaccination broke the mould: a novel trial design involving ring vaccination with recombinant vesicular stomatitis virus vaccine given to contacts of cases demonstrated high efficacy in Guinea, ${ }^{22}$ allowing this vaccine to be deployed in DRC in 2018. The need for rapid vaccine development led to the formation of the Coalition for Epidemic Preparedness Innovations, a public-private partnership which is funding the development of vaccines for MERS-CoV, LF and Nipah viruses.

The trial of novel therapeutics is now being considered during outbreaks where no effective treatment exists. WHO has developed an ethical framework 'Monitored Emergency Use of Unregistered Interventions' and currently two antivirals and three monoclonal-antibody cocktails are approved for use in Ebola in DRC. Trial designs during emergency response have been carefully critiqued and novel randomisation strategies developed. ${ }^{23}$ Excellent basic clinical care is challenging to deliver in viral haemorrhagic fever outbreaks but remains essential for reducing mortality. Well-designed isolation centres, near-patient monitoring devices and portable, rapid biochemistry analysers have revolutionised this.

Innovation in personal protective equipment (PPE) proliferated during the WA Ebola outbreak. Rapid removal PPE suits (www.youtube.com/watch?time_continue=7\&v=kRab2bGahCE), improved fabrics and simulation mannequins have been created, enhancing our ability to safely deliver care.

\section{Conclusion}

Outbreak response has made significant and impressive progress involving a wider range of disciplines, embracing modern technology, and recognising the importance of research during and between outbreaks. More progress is needed and requires that funders and governments recognise the inevitability of the next outbreak of a disease of high consequence and our global vulnerability to it.

\section{References}

1 Al-garadi MA, Khan MS, Varathan KD, Mujtaba G, Al-Kabsi AM. Using online social networks to track a pandemic: A systematic review. J Biomed Inform 2016;62:1-11.

2 Brownstein JS, Freifeld CC. HealthMap: the development of automated real-time internet surveillance for epidemic intelligence. Wkly releases 2007;12:3322.

3 eHealth Africa. Public emergency management disease surveillance - case study - Auto-Visual AFP Detection and Reporting (AVADAR). eHealth Africa. www.ehealthafrica.org/avadar.

4 Calba C, Goutard FL, Hoinville L et al. Surveillance systems evaluation: a systematic review of the existing approaches. BMC Public Health 2015;15:448.

5 Grard G, Fair JN, Lee D et al. A novel rhabdovirus associated with acute hemorrhagic fever in central Africa. PLOS Pathog 2012;8:e1002924.

6 Paweska JT. Lujo virus hemorrhagic fever. In: Ergonul O, Can F, Akova M, Madoff L (eds), Clinical case study of emerging infectious diseases. London: Elsevier/Academic Press, 2014:95-110.

7 Hsu CH, Champaloux SW, Keïta S et al. Sensitivity and specificity of suspected case definition used during west Africa Ebola epidemic. Emerg Infect Dis 2018;24:9-14.

8 Heitzinger K, Impouma B, Farham BL et al. Using evidence to inform response to the 2017 plague outbreak in Madagascar: a view from the WHO African Regional Office. Epidemiol Infect 2018;147:1-5.

9 Fähnrich C, Denecke K, Adeoye OO et al. Surveillance and outbreak response management system (SORMAS) to support the control of the Ebola virus disease outbreak in west Africa. Euro Surveill 2015;20:21071.

10 Sacks JA, Zehe E, Redick C et al. Introduction of mobile health tools to support Ebola surveillance and contact tracing in Guinea. Glob Heal Sci Pract 2015;3:646-59.

11 Tom-Aba D, Olaleye A, Olayinka AT et al. Innovative technological approach to Ebola virus disease outbreak response in Nigeria using the open data kit and form hub technology. PLoS One 2015;10:e0131000.

12 Semper AE, Broadhurst MJ, Richards ] et al. Performance of the GeneXpert Ebola assay for diagnosis of Ebola virus disease in Sierra Leone: a field evaluation study. PLOS Med 2016;13:e1001980.

13 Jean Louis F, Huang JY, Nebie YK et al. Implementation of broad screening with Ebola rapid diagnostic tests in Forécariah, Guinea. Afr J Lab Med 2017;6:1-6.

14 Broadhurst MJ, Kelly JD, Miller A et al. ReEBOV antigen rapid test kit for point-of-care and laboratory-based testing for Ebola virus disease: a field validation study. Lancet 2015;386:867-74.

15 Mead PS. Plague in Madagascar - a tragic opportunity for improving public health. N Engl J Med 2018;378:106-8.

16 Fund The Global. Rebuilding health care in the shadow of Ebola the global fund to fight aids, tuberculosis and malaria. Voices 2017. www.theglobalfund.org/en/blog/2017-02-20-rebuilding-health-carein-the-shadow-of-ebola.

17 WHO Ebola Response Team. Ebola virus disease in west Africa - The first 9 months of the epidemic and forward projections. N Engl J Med 2014;371;1481-95.

18 Pandey A, Atkins KE, Medlock ] et al. Strategies for containing Ebola in west Africa. Science 2014:346:991-5.

19 Lourenço J, Maia de Lima M, Faria NR et al. Epidemiological and ecological determinants of Zika virus transmission in an urban setting. Elife 2017;6:e29820. 
20 Faria NR, Sabino EC, Nunes MR et al. Mobile real-time surveillance of Zika virus in Brazil. Genome Med 2016;8:97.

21 von Seidlein L, Kekulé AS, Strickman D. Novel vector control approaches: the future for prevention of Zika virus transmission? PLOS Med 2017;14:e1002219.

22 Henao-Restrepo AM, Longini IM, Egger M et al. Efficacy and effectiveness of an rVSV-vectored vaccine expressing Ebola surface glycoprotein: interim results from the Guinea ring vaccination cluster-randomised trial. Lancet 2015;386:857-66.
23 Brueckner M, Titman A, Jaki T, Rojek A, Horby P. Performance of different clinical trial designs to evaluate treatments during an epidemic. PLoS One 2018;13:e0203387.

Address for correspondence: Dr Catherine Houlihan, London School of Hygiene and Tropical Medicine, Keppel Street, London WC1E 7HT, UK.

Email: catherine.houlihan@lshtm.ac.uk.

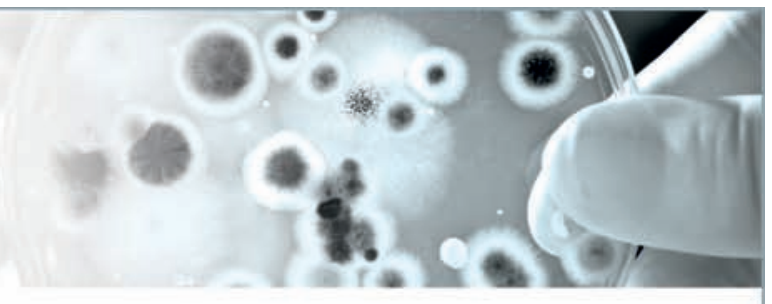

Medical Care

\section{Are you involved in planning infectious diseases and tropical medicine services?}

Medical Care is the online evolution of the well-known RCP publication Consultant physicians working with patients. Written by leading medical specialty experts, it is a comprehensive web-based resource for the efficient and effective design of medical services covering all the specialties, including infectious diseases and tropical medicine.

\section{www.rcpmedicalcare.org.uk}

The resource looks in detail at the services delivered by infectious diseases and tropical medicine, including:

$>$ inpatient services

$>$ dinics

> outpatient parenteral antibiotic therapy services

$>$ refugee and asylum seeker health services

$>$ travel health services.

Medical Care has been designed to help those involved in the planning and provision of medical services to get a clearer picture of the specialty services that need to be in place to provide great patient care. 